Brief communication

\title{
Quality of life of lung cancer patients: Validation of the Taiwan Chinese version of the EORTC QLQ-C30 and QLQ-LC13
}

\author{
Wei-Chu Chie ${ }^{1}$, Chih-Hsin Yang ${ }^{2}$, Chiun Hsu ${ }^{2}$ \& Pan-Chyr Yang ${ }^{3}$ \\ ${ }^{1}$ School of Public Health and Graduate Institute of Preventive Medicine, College of Public Health, National \\ Taiwan University (E-mail: weichu@episerv.cph.ntu.edu.tw); ${ }^{2}$ Department of Oncology; ${ }^{3}$ Internal Medicine, \\ National Taiwan University Hospital, Taiwan
}

Accepted in revised form 24 December 2002

\begin{abstract}
The purpose of this study was to test the validity and reliability of the Taiwan Chinese translation of the European Organization for Research and Treatment of Cancer Quality of Life Questionnaire Core-30 (EORTC QLQ-C30, version 3) and Quality of Life Questionnaire Lung Cancer-13 (QLQ-LC13) questionnaires. Consecutively 51 patients with lung cancer undergoing active chemotherapy and 48 such patients undergoing regular follow-up completed the questionnaires. The intraclass correlation between test and retest ranged from 0.46 to 0.85 for the QLQ-C30 and was 0.76 for dyspnea for the QLQ-LC13. The $\kappa$ coefficients between test and retest ranged from 0.51 to 0.73 for single items of the QLQ-C30 and 0.49-0.68 for five of the nine items in the QLQ-LC13. The Cronbach's $\alpha$ coefficients were $\geqslant 0.70$ for all scales of the two questionnaires apart from that of cognitive functioning. The correlation coefficients between indices measuring similar dimensions of the EORTC QLQ-C30 and the SF-36 questionnaires ranged from 0.43 to 0.73, and that between the dyspnea scales of the two EORTC questionnaires was 0.70. Patients in the follow-up group revealed higher scores of global status/quality of life, and lower scores of nausea/vomiting, as also physical functioning. The questionnaires could also detect expected adverse effects of radiotherapy, cisplatin, and paclitaxel.
\end{abstract}

Key words: EORTC QLQ-C30, EORTC QLQ-LC13, Lung cancer, Quality of life

\section{Introduction}

Lung cancer is a disease that is rapidly increasing in Taiwan [1]. The high case-fatality rate (95.7\%) [1] spurred oncologists to develop new therapeutic measures to prolong lung cancer patients' life [2].

More than 50 instruments measuring quality of life (QOL) for patients suffering from lung cancer emerged from 1970 to 1995 [3]. The Lung Cancer Symptom Scale (LCSS), Functional Assessment of Cancer Therapy-Lung (FACT-L) and the European Organization for Research and Treatment of Cancer Quality of Life Questionnaire Core-30 (EORTC QLQ-C30) with Quality of Life Questionnaire Lung Cancer-13 (QLQ-LC13) have good reliability and validity [3]. The EORTC QLQ-C30 includes 30 questions that measure five function scales, three symptom scales, five single symptom items and financial difficulty. All scales/items are transformed to scores ranging between 0 and 100 . For all functioning scales that measure function a higher score represents better QOL; and for all symptom scales/items a lower score means better QOL. Previous studies using this instrument revealed high internal consistency in most scales except for cognitive functioning, good inter-scale correlation, good discriminant validity [4], testretest reliability [5], patient-observer agreement [6] and cross-cultural validity except a low discriminant validity of role functioning scale in Japan [7]. 
The QLQ-LC13 is a supplementary module to the QLQ-C30 for patients with lung cancer and contains 13 questions to measure one symptom scale (dyspnea) and nine single symptom items. A previous study demonstrated good internal consistency for the dyspnea scale and good clinical validity for most scores [8].

The only available Chinese translation prior to this study, the Cantonese version of the EORTC QLQ-C30 [9] included vocabulary that differed from the Mandarin typically used in Taiwan. The Standard Chinese version of the QLQ-C30 (version 2) was available in 1997 and the results of validation were published in 2000 [10]. As a result of the geographical, political and cultural separation of China and Taiwan for over 50 years, each country's corresponding languages and alphabetical characters have evolved and become somewhat different. Here we present the result of translation and cross-validation of the EORTC QLQ-C30 version 3 and QLQ-LC13 questionnaires for lung cancer patients in Taiwan.

\section{Methods}

We followed the EORTC guidelines for the translation procedure and pilot testing of the questionnaire [11]. The Taiwan Standard version (version 1.0) of the SF-36 questionnaire, a generic instrument measuring eight separate dimensions of QOL [12, 13] was used as a comparison instrument. The Taiwan Standard version (version 1.0) of the SF-36 questionnaire has demonstrated good reliability and validity in Taiwan [14-17].

We consecutively contacted lung cancer patients undergoing active chemotherapy and undergoing post-therapy follow-up at the out patient clinic of the Department of Oncology of the National Taiwan University Hospital from November 2000 to December 2001 inclusively. Chemotherapy and the concurrent medical treatment data were also collected on patients undergoing active treatment. Written consent was obtained from all study participants. Patients who declined to participate, or who had participated in other studies or who had elected to amend their treatment plans were excluded. Assuming that the standard deviation of the questionnaire-derived QOL score is 20, the sample size required for each group is 50 subjects in order to achieve $80 \%$ power for detecting a difference of 10 points between two groups at the $5 \%$ significant level for a one-sided $Z$-test. The final participant distribution was 51 for the active treatment group and 48 for the follow-up group after correcting for misclassified patients and the excluding non-eligible patients. The selection of the two known groups was based upon the hypotheses that patients undergoing chemotherapy typically revealed poorer QOL.

Patients were instructed to complete the questionnaires themselves. However, four patients in the active treatment group and seven patients in the follow-up group who were illiterate were assisted by two interviewers fluent in both Mandarin and Taiwanese. The illiterate subjects were kept in the study to maintain the representativeness of the sample. Patients in the follow-up group were retested with the same questionnaires 1-2 weeks after the first test, conducted by telephone interview or by mailing the questionnaires. Test/retest reliability of the translated questionnaire was carried out on the follow-up group since their medical condition was expected to be more stable than that of patients in active treatment.

Answers to the three questionnaires were scored according to the instructions and computer programs provided $[12,13,18]$. The test/retest reliability was evaluated by intraclass correlation coefficients (ICCs) for each questionnaire-determined dimension, and $\kappa$ value of two repeated tests for single items. Cronbach's $\alpha$ coefficient was used in order to evaluate the internal consistency of each dimension. The validity of the two questionnaires was examined by Pearson's correlation coefficients between similar dimensions for the EORTC and the SF-36 questionnaires. The Wilcoxon's rank sum test was used to examine the difference in scores for each dimension, and the Cochran Mantel-Haenszel test was used to examine differences in single items between the two groups of patients. A $p$-value of $<0.05$ was considered as statistically significant.

\section{Results}

Patients in the active chemotherapy group $(n=51)$ averaged 54.3 years of age $(\mathrm{SD}=12.1$ years), included more men (32) than women, had about the 
same number with less than a high school education (22) as with a high school education or better (29), spoke mostly Mandarin (39) or Taiwanese (39) and had adenocarcinoma or squamous cell cancer. Patients in the follow-up group averaged 55.0 years of age ( $\mathrm{SD}=12.4$ years), included almost equal numbers of men and women, had the same number with less than or at least a high school education (24), spoke mostly Taiwanese (38) and had mostly adenocarcinoma (32).

The ICCs were moderate to high in most scales $(r=0.46-0.85$ for the QLQ-C30 and 0.76 for dyspnea of the QLQ-LC13). The $\kappa$ coefficients for and single items were moderate for the QLQ-C30 $(\kappa=0.51-0.73)$ and also for five items in the QLQLC13 ( $\kappa=0.49-0.68)$, but low for the remaining four items of the QLQ-LC13 (hemoptysis, peripheral neuropathy, pain in arm and pain in other part, $\kappa=0.35-0.39)$. All Cronbach's $\alpha$ coefficients for scales of the QLQ-C30 and the dyspnea scale of the QLQ-LC13 were satisfactory $(\geqslant 0.70)$ apart from that coefficient for cognitive functioning $(\alpha=0.58)$. The Cronbach's $\alpha$ coefficient of painrelated questions was only 0.58 , while that for peripheral neuropathy plus pain questions was 0.69 (Table 1).

The physical functioning, emotional functioning and pain scales of the QLQ-C30 were highly correlated $(r \geqslant 0.70)$ with scales measuring the same domains in the SF-36. The dyspnea scale of the QLQ-LC13 was highly correlated $(r=0.70)$ with the single item of dyspnea (short of breath) of the QLQ-C30. Correlation coefficients between the remaining pairs of scales were moderate $(r=0.43$ $0.63)$. The follow-up group demonstrated a higher score for the QOL scale, a lower score for the

Table 1. Reliability of the EORTC QLQ-C30 and the EORTC QLQ-LC13

\begin{tabular}{|c|c|c|}
\hline & $\begin{array}{l}\text { Test-retest reliability } \\
\text { (Follow-up group, } \mathrm{N}=48 \text { ) }\end{array}$ & $\begin{array}{l}\text { Internal consistency } \\
\text { (All patients, } N=99 \text { ) }\end{array}$ \\
\hline \multicolumn{3}{|l|}{$Q L Q-C 30$} \\
\hline Intraclass correlation coefficients & & Cronbach's $\alpha$ \\
\hline Physical functioning & 0.85 & 0.85 \\
\hline Role functioning & 0.58 & 0.92 \\
\hline Emotional functioning & 0.76 & 0.81 \\
\hline Cognitive functioning & 0.83 & 0.58 \\
\hline Social functioning & 0.46 & 0.82 \\
\hline QOL & 0.75 & 0.86 \\
\hline Pain & 0.46 & 0.80 \\
\hline Nausea and vomiting & 0.52 & 0.74 \\
\hline Fatigue & 0.64 & 0.81 \\
\hline \multicolumn{3}{|l|}{ Agreement \% $(\kappa)$} \\
\hline Dyspnea & $71 \%(0.53)$ & - \\
\hline Sleep disturbance & $77 \%(0.67)$ & - \\
\hline Appetite loss & $79 \%(0.66)$ & - \\
\hline Constipation & $79 \%(0.53)$ & - \\
\hline Diarrhea & $79 \%(0.51)$ & - \\
\hline Financial difficulty & $85 \%(0.73)$ & - \\
\hline \multicolumn{3}{|l|}{$Q L Q-L C 13$} \\
\hline Intraclass correlation coefficients & & Cronbach's $\alpha$ \\
\hline Dyspnea & 0.76 & 0.83 \\
\hline \multicolumn{3}{|l|}{ Agreement \% (к) } \\
\hline Coughing & $77 \%(0.62)$ & - \\
\hline Hemoptysis & $92 \%(0.39)$ & - \\
\hline Sore mouth & $85 \%(0.49)$ & - \\
\hline Dysphagia & $83 \%(0.51)$ & - \\
\hline P. neurophathy & $63 \%(0.38)$ & Pains + this item: 0.69 \\
\hline Alopecia & $83 \%(0.68)$ & - \\
\hline Pain in chest & $75 \%(0.58)$ & All pains: 0.58 \\
\hline Pain in arm & $63 \%(0.39)$ & - \\
\hline Pain others & $65 \%(0.35)$ & - \\
\hline
\end{tabular}


Table 2. Comparison of QOL scores of each scale in the EORTC QLQ-C30 and QLQ-LC13 among two groups of patients

\begin{tabular}{llll}
\hline & $\begin{array}{l}\text { Active chemotherapy } \mathrm{N}=51 \\
\text { Mean } \pm \mathrm{SD}\end{array}$ & $\begin{array}{l}\text { Follow-up N }=48 \\
\text { Mean } \pm \mathrm{SD}\end{array}$ & $\begin{array}{l}\text { Wilcoxon's test } \\
p \text {-value (one-side) }\end{array}$ \\
\hline Physical functioning & $79.9 \pm 18.3$ & $72.2 \pm 22.1$ & $0.0262^{*}(\mathrm{~T}>\mathrm{F})$ \\
Role functioning & $71.6 \pm 25.9$ & $74.7 \pm 31.5$ & $\mathrm{NS}$ \\
Emotional functioning & $75.8 \pm 16.1$ & $75.0 \pm 21.0$ & $\mathrm{NS}$ \\
Cognitive functioning & $80.4 \pm 18.8$ & $78.8 \pm 22.0$ & $\mathrm{NS}$ \\
Social functioning & $69.3 \pm 28.4$ & $76.7 \pm 21.4$ & $\mathrm{NS}$ \\
QOL & $56.5 \pm 19.6$ & $63.2 \pm 20.8$ & $0.0449^{*}(\mathrm{~F}>\mathrm{T})$ \\
Fatigue & $39.2 \pm 21.6$ & $34.3 \pm 19.7$ & $\mathrm{NS}$ \\
Nausea and vomiting & $17.6 \pm 21.7$ & $5.6 \pm 11.1$ & $0.0004(\mathrm{~T}>\mathrm{F})$ \\
Pain & $24.8 \pm 24.8$ & $19.4 \pm 18.3$ & $\mathrm{NS}$ \\
Dyspnea & $20.3 \pm 18.3$ & $24.1 \pm 21.8$ & $\mathrm{NS}$ \\
\hline
\end{tabular}

$* p<0.05$, one-sided.

nausea/vomiting scale, and a lower score for physical functioning scale than the active chemotherapy group. Scores of the two groups did not appear to differ for any other dimensions (Table 2) or single items (not shown in the table). Compared wit patients who did not have the following treatments, patients who underwent radiotherapy and who received cisplatin experienced more nausea and vomiting, patients who received radiotherapy experienced more hair loss and arm/ shoulder pain, and patients who received paclitaxel revealed greater level of hair loss (Table 3).

\section{Discussion}

The test/retest reliabilities of the questionnaires for the follow-up group in this study were lower than was the case for the 1995 study of Hjermstad et al. [5] but consistent with our previous study on patients with nasopharyngeal carcinoma [19]. Our test/retest interval was within the range suggested by Streiner and Norman [20]. It is possible that a patient's condition may have changed within the range even for members in the follow-up group. The results for internal consistency in this study

Table 3. Differences in nausea/vomiting scores of patients with and without certain treatments (only significant results were included)

\begin{tabular}{lrrl}
\hline & N & Mean \pm SD & $p$-value \\
\hline $\begin{array}{l}\text { Nausea and vomiting } \\
\text { Radiotherapy }\end{array}$ & & Wilcoxon's test $p$-value (one-side) \\
$\quad$ Yes & 9 & $25.9 \pm 20.6$ & 0.0490 (Yes $>$ no) \\
$\quad$ No & 42 & $15.9 \pm 21.8$ & \\
Cisplatin & 28 & $23.8 \pm 25.0$ & 0.0161 (Yes $>$ no) \\
$\quad$ Yes & 23 & $10.1 \pm 14.0$ & Cochran Mantel-Haenszel test \\
$\quad$ No & & & 0.0292 \\
Hair loss & 9 & & \\
Radiotherapy & 42 & $33.3 \pm 37.3$ & 0.0059 \\
$\quad$ Yes & $11.1 \pm 24.0$ & \\
$\quad$ No & 10 & $36.7 \pm 39.9$ & Cochran Mantel-Haenszel test \\
Paclitaxel & 41 & $9.8 \pm 21.4$ & 0.0150 \\
$\quad$ Yes & & & \\
$\quad$ No & & $44.4 \pm 28.9$ & \\
Arm and shoulder pain & 9 & $19.8 \pm 25.6$ & \\
Radiotherapy & 42 & & \\
$\quad$ Yes & & & \\
$\quad$ No & & & \\
\hline
\end{tabular}


were consistent with the findings of previous studies $[4,7,8,10]$. We changed the translation peripheral neuropathy (tingling) from 'pain as having been stung' to 'like having been stung with needles' because of the unexpectedly high Cronbach's $\alpha$ coefficient of tingling and pain questions.

The moderate to high correlation of similar dimensions between the QLQ-C30 and the SF-36 and the high correlation between dyspnea scales of the two EORTC questionnaires imply that both EORTC questionnaires exhibited good validity. The scores for the active chemotherapy group of this study were similar to or better than for those of patients during treatment in previous studies [4, $8,10]$. The scores for the follow-up group of this study were similar to the reference values of local or locoregional non-small cell lung cancer provided by the EORTC [21], but better than that of previous studies $[4,7]$. The significantly higher scores in the QOL and lower score in the nausea/ vomiting scale of the follow-up group corresponded with the finding of Bergmen et al. [8]. The corresponding symptoms of cisplatin (nausea/ vomiting), paclitaxel (hair loss), and radiotherapy (shoulder/arm pain) were consistent with previous knowledge of the adverse effects. The pronounced hair loss experienced by patients undergoing radiotherapy was, however, difficult to explain and warrants further investigation.

\section{Acknowledgments}

This study was supported by a two-year research grant provided by the National Science Council, Taiwan, no. NSC89-2314-B002-469 and no. NSC89-2320-B-002-096. The authors of this study are grateful to Ms Karen West for her professional help in translation review, Drs Pei-Ching Oon and Fong Liew, Mr Wei-Hua Tan and Mr Jui-Ming Shih for translation, Professor Jen-pei Liu for statistical consultation, as well as Dr Chia-Chi Lin, Ms Chia-Chun Lai, and Mr Ming-Jer Chang for assistance with data collection.

\section{References}

1. Department of Health, the Executive Yuan, Cancer Registry Annual Report, Republic of China, 1998. Web-site of Department of Health: www.doh.gov.tw, 2002.
2. Division of Cancer Research, National Health Research Institutes and Taiwan Cooperative Organization of Cancer. Consensus of Diagnosis and Treatment of Lung Cancer. Taipei: National Health Research Institutes, 1998.

3. Montazeri A, Gills CR, McEwen J. Quality of life in patients with lung cancer. Chest 1998; 113: 467-481.

4. Aaronson NK, Ahmedzai A, Berman B, et al. The European Organization for Research and Treatment of Cancer QLQ-C30: A quality-of-life instrument for use in international clinical trials in oncology. J Natl Cancer Inst 1993; 85: 365-376.

5. Hjerstad MJ, Fossa SD, Bjordal K, Kaasa S. Test/retest study of the European Organization for Research and Treatment of Cancer Core Quality-of-Life Questionnaire. J Clin Oncol 1995; 13: 1249-1254.

6. Groenvold M, Klee MC, Sprangers MAG, Aaronson NK. Validation of the EORTC QLQ-C30 quality of life questionnaire through combined qualitative and quantitative assessment of patient-observer agreement. J Clin Epidemiol 1997; 50: 441-450.

7. Kobayashi K, Takeda F, Teramukai S, et al. A cross-validation of the European Organization for Research and Treatment of Cancer QLQ-C30 (EORTC QLQ-C30) for Japanese with lung cancer. Eur J Cancer 1998; 34: 810-815.

8. Bergman B, Aaronson NK, Ahmedzai S, et al. The EORTC QLQ-C13: A modular supplement to the EORTC Core Quality of Life Questionnaire (QLQ-C30) for use in lung cancer clinical trials. Eur J Cancer 1994; 30A: 635-642.

9. EORTC quality of Life Study Group. Questionnaire for the Department of Obstetric and gynecology, Hong Kong University (Chinese Translation of the EORTC QLQ-C30 version 2.0). Brussels: Quality of Life Unit, EORTC Data Center, 1997.

10. Zhao H, Kanada K. Translation and validation of the Standard Chinese version of the EORTC QLQ-C30. Qual Life Res 2000; 9: 139-137.

11. Cull A, Sprangers M, Bjordal K, et al. EORTC Quality of Life Study Group Translation Procedure. Brussels: EORTC Quality of Life Study Group, 1998.

12. New England Medical Center Hospital. IQOLA SF-36 Taiwan Standard Version 1.0. Boston: The Health Institute, New England Medical Center, 1996.

13. Ware JE, Kosinski M, Keller SD. SF-36 Physical and Mental Health Summary Scales: A User's Manual. Boston: The Health Institute, New England Medical Center, 1994.

14. Chie WC, Huang CS, Chen JH, Chang KJ. Measurement of the quality of life during different clinical phases of breast cancer. J Formos Med Assoc 1999; 98: 254-260.

15. Fuh JL, Wang SJ, Lu SR, Juang KD, Lee SJ. Psychometric evaluation of a Chinese (Taiwanese) Version of the SF-36 Health Survey amongst middle-aged women from a rural community. Qual Life Res 2000; 9: 675-683.

16. Chiu HC, Chern JY, Shi HY, Chen SH, Chang JK. Physical functioning and health-related quality of life: Before and after total hip replacement. Kaohsiung J Med Sci 2000; 16 : 285-292.

17. Wang SJ, Fuh JL, Lu SR, Juang KD. Quality of life differs among headache diagnoses: Analysis of SF-36 survey in 901 headache patients. Pain 2001; 89: 285-292. 
18. Fayers P, Aaronson N, Bjordal K, Curran D, Groenvold M. EORTC QLQ-C30 Scoring Manual. 2nd ed. Brussels: EORTC Quality of Life Study Group, 1999.

19. Chie WC, Hong RL, Lai CC, Ting LL, Hsu MM. Quality of life in patients of nasopharyngeal carcinoma: Validation of the Taiwan Chinese version of the EORTC QLQ-C30 and the EORTC QLQ-H\&N35. Qual Life Res 2003; 12: 93-98.

20. Streiner DL, Norman GR. Reliability. In Streiner DL, Norman GR (eds). Health Measurement Scales. A Practical Guide to Their Development and Use. Oxford: Oxford Medical, 1994; 79-96.
21. Fayers P, Weeden S, Curran D. EORTC QLQ-C30 Reference Values. Brussels: EORTC Quality of Life Study Group, 1998.

Address for correspondence: Wei-Chu Chie, School of Public Health and Graduate Institute of Preventive Medicine, College of Public Health, National Taiwan University, Room 209, 19 Hsuchow Road, Taipei 10020, Taiwan

Phone: + 886-2-23920460; Fax: +886-2-23920456

E-mail: weichu@episerv.cph.ntu.edu.tw 